\title{
BMJ Open Reference ranges of fetal heart function using a Modified Myocardial Performance Index: a prospective multicentre, cross-sectional study
}

\begin{tabular}{l}
\hline Lijuan Sun (1),${ }^{1}$ Jingjing Wang, ${ }^{1}$ Xiaoting Su, ${ }^{2}$ Xinlin Chen, ${ }^{3}$ Yuqing Zhou, ${ }^{4}$ \\
Xiaoming Zhang, ${ }^{5}$ Hong Lu, ${ }^{6}$ Jianmei Niu, ${ }^{7}$ Lan Yu, ${ }^{8}$ Congxin Sun, \\
Wenjun Zhang, ${ }^{10}$ Jijing Han, ${ }^{1}$ Lina Zhang, ${ }^{1}$ Zhenna Wang, ${ }^{2}$ Peiwen Chen, ${ }^{3}$ \\
Tiantian Chen, ${ }^{4}$ Hua Hong, ${ }^{5}$ Lulu Zhou, ${ }^{6}$ Baoying Ye, ${ }^{7}$ Wei Guo, ${ }^{8}$ Wei Zhao, \\
Na Zhang, ${ }^{1}$ Zhen Li, ${ }^{1}$ Sheng Zhao, ${ }^{3}$ Qingqing Wu
\end{tabular}

To cite: Sun L, Wang J, Su X, et al. Reference ranges of fetal heart function using a Modified Myocardial Performance Index: a prospective multicentre, crosssectional study. BMJ Open 2021;11:e049640. doi:10.1136/ bmjopen-2021-049640

- Prepublication history for this paper is available online. To view these files, please visit the journal online (http://dx.doi. org/10.1136/bmjopen-2021049640).

Received 31 January 2021 Accepted 27 May 2021

Check for updates

(c) Author(s) (or their employer(s)) 2021. Re-use permitted under CC BY-NC. No commercial re-use. See rights and permissions. Published by BMJ.

For numbered affiliations see end of article.

Correspondence to Dr Qingqing Wu; qingqingwu@ccmu.edu.cn, Dr Jiawei Tian; jwtian2004@163.com and Dr Yuxin Jiang; jiangyuxinxh@163.com

\section{ABSTRACT}

Objectives The primary aim of this study was to establish the normal reference ranges of the fetal left ventricular (LV) Modified Myocardial Performance Index (Mod-MPI). A secondary aim was to evaluate the agreement between manual and automatic measurements for fetal Mod-MPI. Design A prospective, multicentre, cross-sectional study. Participants Normal singleton pregnancies.

Methods The LV functions of normal singleton pregnancies were assessed in nine centres covering eight provinces in China using unified ultrasound protocols and settings and standardised measurements by pulsed Doppler at 20-24, 28-32 and 34-38 weeks of gestation. The isovolumetric relaxation time (IRT), isovolumetric contraction time, ejection time (ET) and Mod-MPI were measured both automatically and manually.

Results This cross-sectional study included 2081 fetuses, and there was a linear correlation between gestational age (GA) and Mod-MPI $(0.416+0.001 \times G A$ (weeks), $p<0.001$, $\left.r^{2}=0.013\right)$, IRT $(36.201+0.162 \times G A$ (weeks), $p<0.001$, $\left.r^{2}=0.021\right)$ and ET (171.418-0.078*GA (weeks), $p<0.001$, $\left.r^{2}=0.002\right)$. This finding was verified using longitudinal data in a subgroup of 610 women. The agreement between the manual and automated measurements for Mod-MPI was good.

Conclusions We constructed normal reference values of fetal LV Mod-MPI. Automatic measurement can be considered for ease of measurement in view of the good agreement between the automatic and manual values.

\section{INTRODUCTION}

The Myocardial Performance Index (MPI or Tei index) is a quantitative tool for evaluating global cardiac function by incorporating the assessment of systolic and diastolic cardiac function. This index was first described in adults by Tei $e t a l$. The modified MPI (ModMPI) can be evaluated using aortic and mitral Doppler valve clicks as landmarks to demarcate the time intervals ${ }^{2}$ after further adjustment. However, the normal reference ranges

\section{Strengths and limitations of this study}

- This is the only large multicentre prospective study from nine centres covering eight provinces and five geographical divisions in China.

- The study used standardised measurements and unified ultrasound settings with well-defined inclusion and exclusion criteria.

- Longitudinal data were used to test and verify the changes in fetal Modified Myocardial Performance Index (Mod-MPI) and time intervals with advanced gestational age.

- Only three time points (20-24, 28-32 and 34-38 weeks of gestation) were set in consideration that left ventricular Mod-MPI showed a slight increase as pregnancy advanced.

of fetal Mod-MPI still show wide variation in recent studies. ${ }^{3-14}$ We have analysed the reasons for this, and they are probably due to a lack of a large enough sample size, a lack of standardised measurement techniques of Mod-MPI, heterogeneous study populations and possibly other maternal or fetal characteristics.

Recently, Mod-MPI has been reported to be a valuable index for evaluating fetal cardiac function and it has been evaluated as an indicator for predicting pregnancy outcomes in a number of studies. ${ }^{15-23}$ Evaluation of ModMPI depends on comparison to a normal reference ranges, so a large multicentre study using standardised machine settings and techniques with well-defined inclusion and exclusion criteria is still required, which forms the basis of this study. The primary aim of this study was to establish the normal reference ranges of fetal left ventricular (LV) Mod-MPI and time intervals by pulsed 
Box 1 Women must have the following characteristics at Booking ( $<14$ weeks of gestation)

a. Aged $\geq 18$ and $<35$ years.

b. Body mass index $\geq 18.5$ and $<30 \mathrm{~kg} / \mathrm{m}^{2}$.

c. Singleton pregnancy.

d. A known last menstrual period with regular cycles (defined as $28 \pm 7$ days) without hormonal contraceptive use, or breast feeding in the 3 months before pregnancy.

e. Natural conception.

f. No relevant medical history, with no need for long-term medication (including fertility treatment and over-the-counter medicines, but excluding routine iron, folate, calcium, iodine or multivitamin supplements).

g. No use of tobacco or recreational drugs such as cannabis in the 3 months before or after becoming pregnant.

h. No heavy alcohol use and caffeine intake(defined as $>5$ units $(50 \mathrm{~mL}$ pure alcohol) per week) since becoming pregnant.

i. No more than one miscarriage in the two previous consecutive pregnancies.

j. No previous baby delivered preterm $\left(<37^{+0}\right.$ weeks of gestation) or with a birth weight $<2500 \mathrm{~g}$ or $>4000 \mathrm{~g}$.

k. No previous neonatal or fetal death, ectopic pregnancy, previous baby with any congenital malformations, and no evidence in present pregnancy of congenital disease or fetal anomaly.

I. No previous pregnancy affected by pre-eclampsia/eclampsia, haemolytic anaemia, elevated liver function and low platelet count syndrome, gestational diabetes mellitus/ gestational impaired glucose tolerance, a related pregnancy-associated condition.

$m$. Normal clinical test indexes.

n. Systolic blood pressure $<140 \mathrm{~mm} \mathrm{Hg}$ and diastolic blood pressure $<90 \mathrm{~mm} \mathrm{Hg}$.

0. Not in an occupation with risk of exposure to chemicals or toxic substances.

p. Early evaluation of gestational age confirmed by ultrasound examination before 14 weeks.

Doppler throughout gestation. A secondary aim was to evaluate the agreement between manual and automatic measurements for fetal Mod-MPI. As the placement of callipers on each valve click is highly dependent on operators, it has been suggested that the Auto Mod-MPI system may increase the reliability and reproducibility, but these conclusions have been based on small sample sizes in previous studies. ${ }^{23} 24$

\section{METHODS}

\section{Design, settings and participants}

This was a multicentre, prospective cross-sectional study (including a subsample of longitudinal data to verify findings from the cross-sectional study) with a large sample size undertaken between 1 August 2015 and 30 June 2019. Nine centres, covering eight provinces in China, participated in the study: Beijing (Beijing Obstetrics and Gynaecology Hospital, Capital Medical University), Hubei (Maternal and Child Health Hospital of Hubei Province), Shandong(Qingdao Women and Children's Hospital), Inner Mongolia Autonomous Region (Inner Mongolia People's Hospital), Xinjiang Uygur Autonomous Region

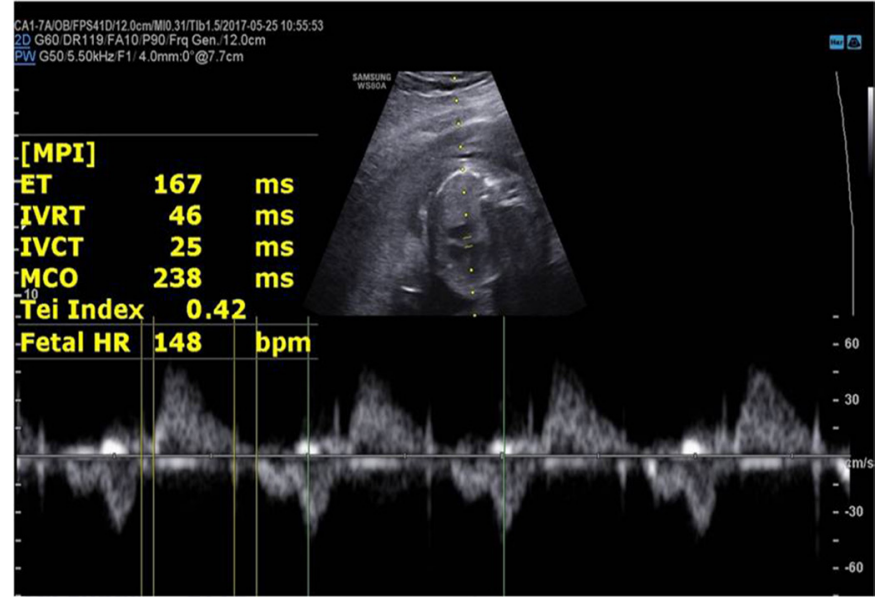

Figure 1 Normal Modified Myocardial Performance Index (Mod-MPI) (Tei index) Doppler image with automatic measurement. $\mathrm{MCO}=$ mitral valve closing and open time $=\mathrm{ICT}$ + IRT+ET. ET, ejection time; ICT, isovolumetric contraction time; IRT, isovolumetric relaxation time.

(People's Hospital of Xinjiang Uygur Autonomous Region), Zhejiang (Women's Hospital School of Medicine Zhejiang University), Shanghai(The International Peace Maternity and Child Health Hospital, School of Medicine, Shanghai Jiao Tong University, and Shanghai Changning Maternity \& Infant Health Hospital), and Hebei (Shijiazhuang Obstetrics and Gynaecology Hospital), which are distributed widely innorthern, central, eastern, northwestern and northeastern China.

Women were eligible to participate in the crosssectional study if they had a normal singleton pregnancy. Women then underwent at least one ultrasound scan at 20-24, 28-32, or 34-38 weeks of gestation. A subgroup of women from the cross-sectional study were also invited to take part in the longitudinal verification study and underwent the entire series of four ultrasound examinations at 11-14, 20-24, 28-32, and 34-38 weeks of gestation.

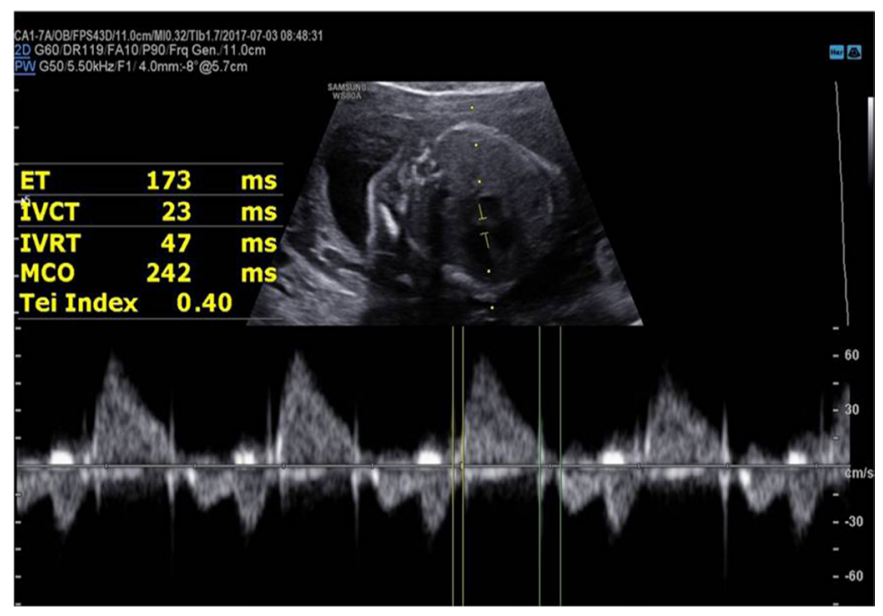

Figure 2 Normal Modified Myocardial Performance Index (Mod-MPI) (Tei index) Doppler image with manual measurement. $\mathrm{MCO}=$ mitral valve closing and open time $=I C T$ + IRT+ET. ET, ejection time; ICT, isovolumetric contraction time; IRT, isovolumetric relaxation time. 


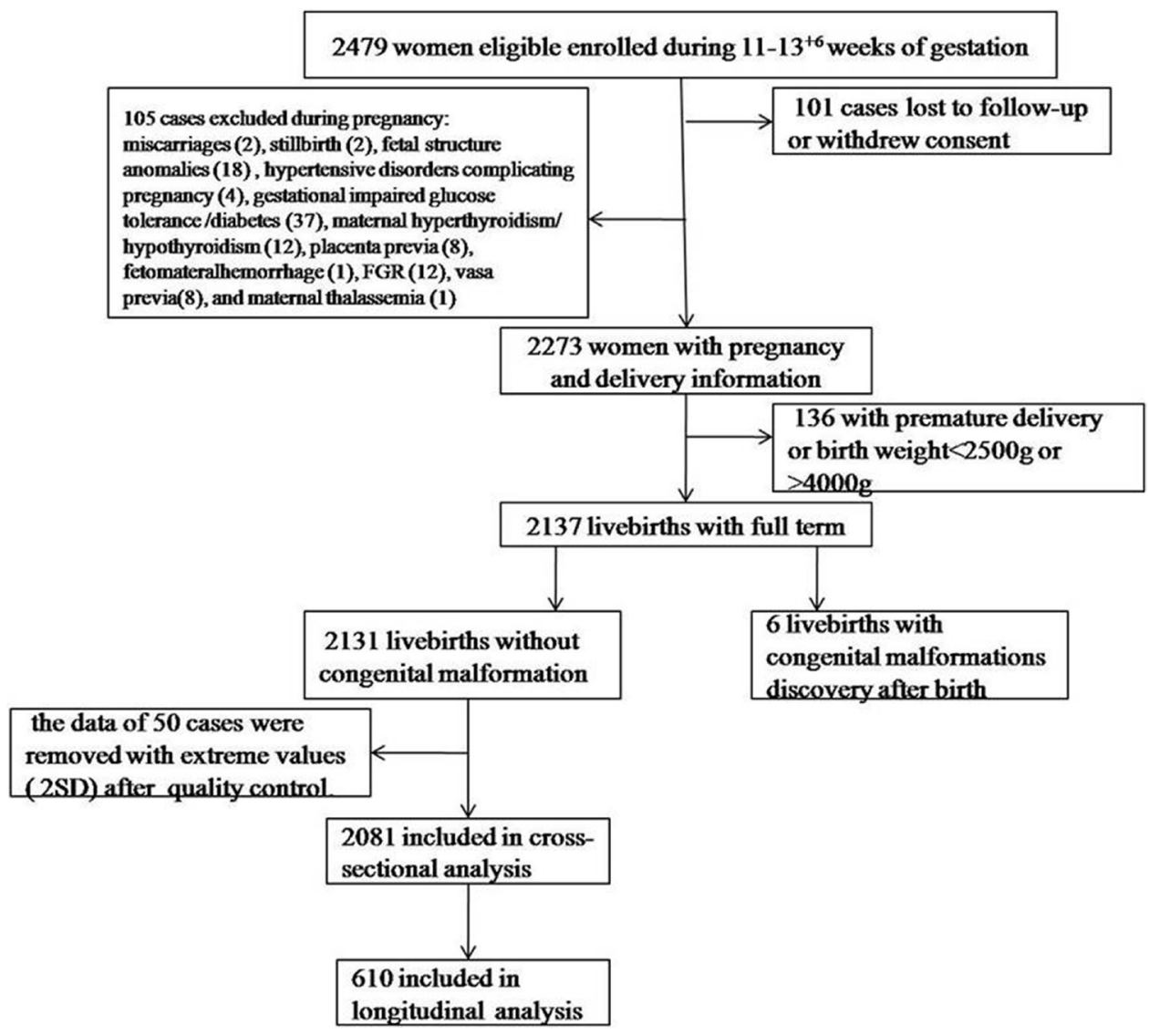

Figure 3 The study flow chart. FGR, fetal growth restriction.

At each hospital, we recruited pregnant women with no clinically relevant obstetric, gynaecological or medical history who initiated antenatal care before 14 weeks of gestation. To approach an optimal health standard, inclusion criteria for the study were based on the fetal Growth Longitudinal Study of INTERGROWTH-21st Project, ${ }^{25}$ with minor modifications of the normal pregnancy conditions, as described in detail in box 1 .

\begin{tabular}{ll}
\hline $\begin{array}{l}\text { Table } 1 \text { Baseline characteristics and pregnancy outcomes } \\
\text { of the study population }\end{array}$ & Pregnancies \\
\hline & $28.6(2.7)$ \\
\hline Maternal age, years & $162.0(4.9)$ \\
\hline Maternal height, $\mathrm{cm}$ & $57.5(6.3)$ \\
\hline Maternal weight, $\mathrm{kg}$ & $21.9(2.2)$ \\
\hline Body mass index, $\mathrm{kg} / \mathrm{m}^{2}$ & $80 \%$ \\
\hline Nulliparous & $93.9 \%$ \\
\hline Ethnicity the Han nationality & $27 \%$ \\
\hline Caesarean section & $49 \%$ \\
\hline Male sex & $3.4(0.3)$ \\
\hline Birth weight, $\mathrm{kg}$ & $39.3(1.1)$ \\
\hline Birth gestational age, week
\end{tabular}

Data are mean (SD) or number (\%).

Maternal baseline characteristics were measured during $11-13^{+6}$ weeks of gestation.
Patients were excluded if any abnormal pregnancyassociated condition developed during follow-up evaluation, such as fetal growth restriction, small-forgestational-age fetus, pre-eclampsia/eclampsia, haemolytic anaemia, elevated liver function and low platelet count syndrome, gestational impaired glucose tolerance or diabetes, intrahepatic cholestasis, miscarriage, stillbirth, fetal structural malformations, chromosomal abnormalities, inflammatory response syndrome, fetal anaemia, low-lying placenta, placental chorioangioma, polyhydramnios or any other fetal or maternal disease conditions throughout pregnancy. All included women were followed up until birth, and all newborns were examined to confirm the absence of any structural anomalies by a postnatal clinical examination. Those with a birth weight $<2500 \mathrm{~g}$ or $>4000 \mathrm{~g}$ and premature birth (defined as birth at less than $37^{+0}$ weeks of gestation) were further excluded.

\section{Study measurements}

Meticulous standardisation of all measurement techniques was implemented across the nine hospitals. All hospitals used a WS80A ultrasound system (Samsung Medison, Korea) with the same settings. All ultrasonographers taking part in this study underwent standardised training, consisting of a clear understanding of the study protocol, training on the use of the machine, standardisation of measurements for the 
Table 2 The intraobserver and interobserver agreement of manual and automated Mod-MPI

\begin{tabular}{|c|c|c|c|}
\hline$\frac{\text { Item }}{\text { ICC }}$ & Number & $\begin{array}{l}\text { Manual Mod- } \\
\text { MPI }\end{array}$ & $\begin{array}{l}\text { Automated } \\
\text { Mod-MPI }\end{array}$ \\
\hline $\begin{array}{l}\text { Intraobserver } \\
\text { ICC }\end{array}$ & 65 & 0.908 & 0.914 \\
\hline $95 \% \mathrm{Cl}$ & & 0.853 to 0.943 & 0.863 to 0.947 \\
\hline$P$ value & & $<0.001$ & $<0.001$ \\
\hline $\begin{array}{l}\text { Interobserver } \\
\text { ICC }\end{array}$ & 65 & 0.879 & 0.902 \\
\hline $95 \% \mathrm{Cl}$ & & 0.809 to 0.925 & 0.845 to 0.939 \\
\hline$P$ value & & $<0.001$ & $<0.001$ \\
\hline
\end{tabular}

ICC, the intraclass correlation coefficient; Mod-MPI, Modified Myocardial Performance Index.

ultrasound parameters, data collection and qualitycontrol measures. Two sets of ultrasonographic images, including the measurements of Mod-MPI and other Doppler indicators and the assessment of fetal growth and development, were handed in by every specially assigned ultrasonographer from nine centres before the beginning of the study. The ultrasonographers were only allowed to participate once the ultrasonographic pictures were examined and approved by the experts organised by the leading centre (Beijing Obstetrics and Gynaecology Hospital, Capital Medical University). The quality-control measures were based on the regular sampling analysis and mid-period checks of the ultrasonographic images from the nine centres.

Gestational age (GA) was based on the last menstrual period and confirmed by ultrasound between 9 weeks and $13^{+6}$ weeks of gestation, provided that the discrepancy was less than 7 days. We then scanned women at 20-24, 28-32 and 34-38 weeks of gestation. The scan consisted of an assessment of fetal growth, the volume of amniotic fluid, the pulsatility index of the umbilical artery, and the fetal anatomy was examined. All ultrasound scans, including measurement of the crown-rump length, biometry and Doppler evaluations, were performed as described by the International Society of Ultrasound in Obstetrics and practice guidelines. ${ }^{26} 27$

To improve the reproducibility, unified machine settings ${ }^{28}$ and standardised measurements ${ }^{2} 12$ of ModMPI across the sites were used. Imaging of the heart was performed with a transverse four-chamber view, in an apical projection, and at the point where the opening
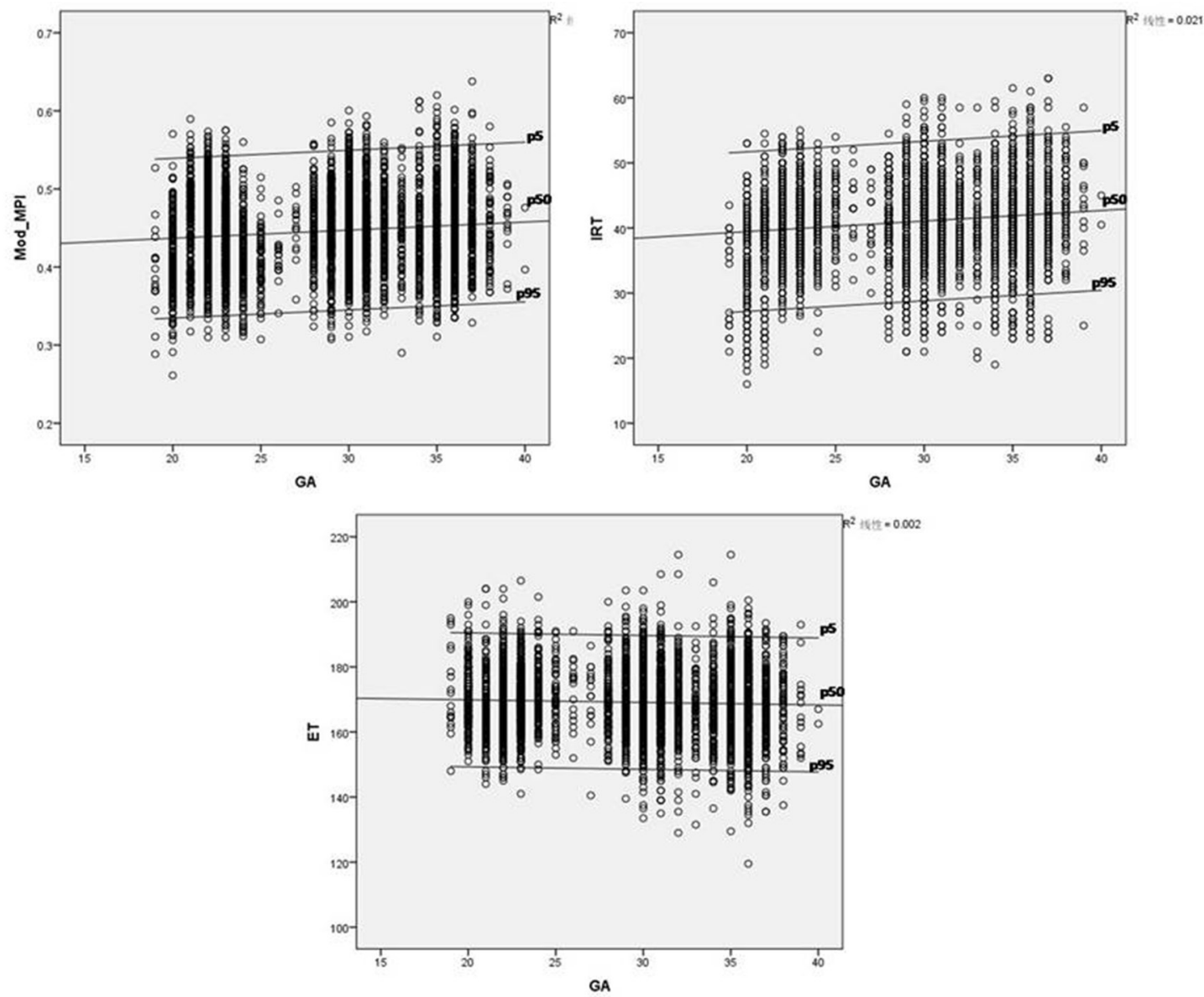

Figure 4 Plots of LV Mod-MPI, IRT and ET based on the cross-sectional data (2081 cases) with advancing gestational age (GA). p5=5th centile; p50=median; p95=95 th centile. ET, ejection time; IRT, isovolumetric relaxation time; Mod-MPI, Modified Myocardial Performance Index. 

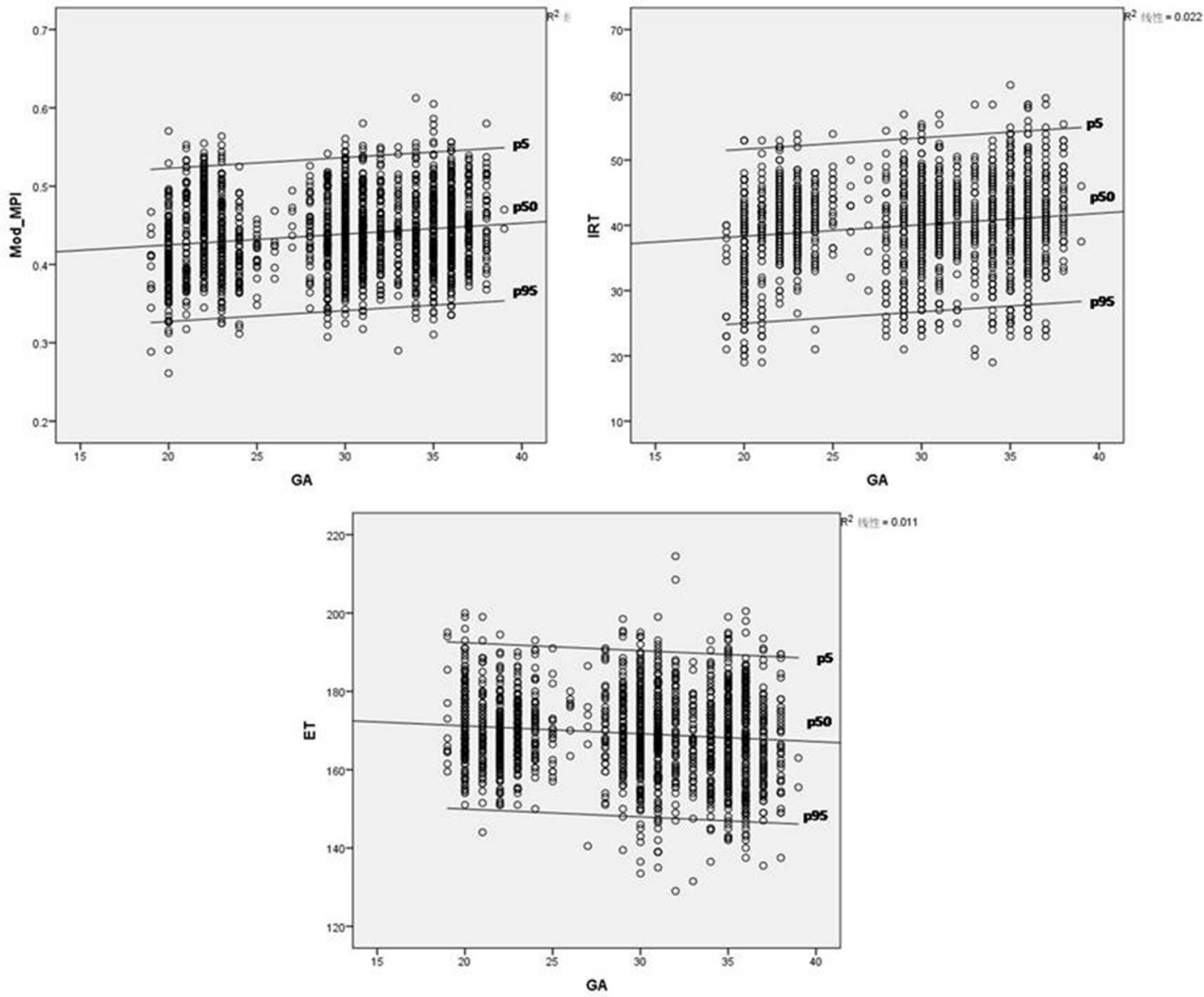

Figure 5 The verification of LV Mod-MPI, IRT and ET with advancing gestational age (GA) based on the longitudinal data (610 cases). p5=5 th centile; p50=median; p95=95 th centile. ET, ejection time; IRT, isovolumetric relaxation time; Mod-MPI, Modified Myocardial Performance Index.

and closure of the mitral and aortic valves were clearly shown. The Doppler sampling gate was placed at the cross between the inflow and outflow of the left ventricle and included the leaflets of the mitral valve (MV) and aortic valve (AV) with a $3-4 \mathrm{~mm}$ gate size ${ }^{4}$ and an insonation angle of less than $15^{\circ}$. The Doppler sweep velocity was set at $11.88-13.67 \mathrm{~cm} / \mathrm{s},{ }^{27}$ the wall motion filter was set at $100 \mathrm{~Hz}$, and the scale was set at $55 \mathrm{~cm} / \mathrm{s}$. Doppler waveforms were obtained during fetal quiescence and after asking the mother to voluntarily suspend respiration. The patients were examined until apical four-chamber views were obtained.

Table 3 The normal reference ranges of fetal left ventricular Mod-MPI based on the cross-sectional data with the manual and automatic measurements

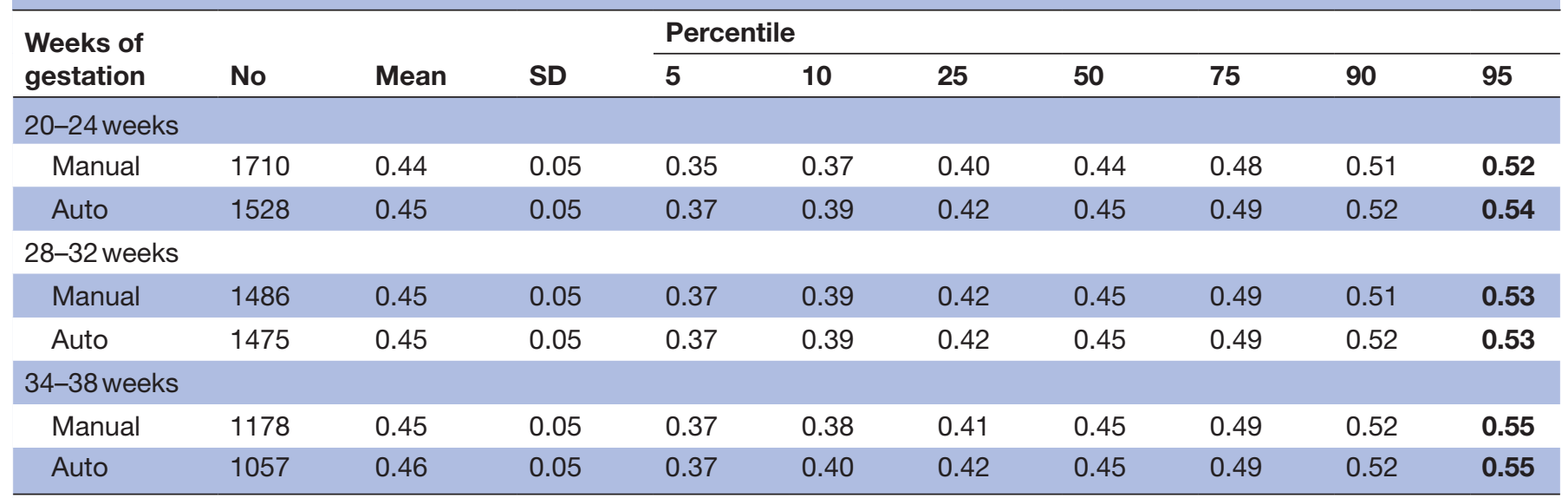

Mod-MPI, Modified Myocardial Performance Index. 
Table 4 The normal reference ranges of fetal left ventricular IRT based on the cross-sectional data with the manual and automatic measurements

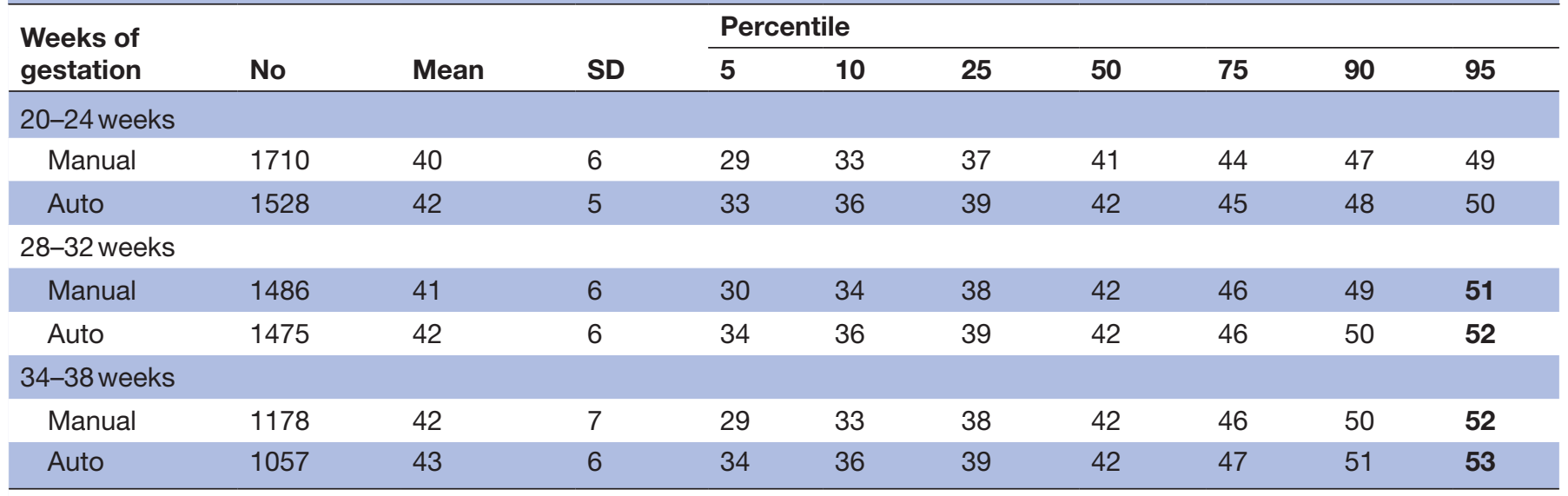

$\mathrm{IRT}$, isovolumetric relaxation time.

We chose the beginning of the valve clicks as the landmark for the measurement period of Mod-MPI to improve reproducibility. ${ }^{1223}$ The callipers were placed just before each valve click (figures 1 and 2).The isovolumetric contraction time (ICT) was measured from closure of the MV to opening of the AV, isovolumetric relaxation time (IRT) from closure of the AV to opening of the MV and ejection time (ET) from opening to closure of the AV. For the automated measurement, the operator placed the cursor on the peak of the aortic outflow of a single Doppler waveform and pressed the 'Set' key, and the Auto Mod-MPI system automatically placed the callipers at the beginning of each valve click. ICT, IRT, ET and Mod-MPI were then calculated automatically. The Mod-MPI was measured twice automatically (figure 1) and twice manually (figure 2). Finally, the mean of the two automatic measurements and the mean of the two manual measurements for each case were used in the statistical analysis.

\section{Follow-up and pregnancy outcome}

Pregnant women and their newborns were followed up until after birth. Any antenatal or intrapartum complications were recorded, and outcomes were ascertained, including GA at birth, delivery mode, birth weight, sex, placental weight and Apgar score.

\section{Patient and public involvement}

Patients and the public were not involved in the design, conduct, reporting, or dissemination plans of our research.

\section{Statistical analysis}

Data were tested for a normal distribution and expressed as the mean \pm SD. The reference values for the 5 th, 10 th, 25th, 50th, 75th, 90th and 95th percentiles for three gestational periods (20-24 weeks, 28-32 weeks and 34-38 weeks) were determined. The intraclass correlation coefficient (ICC) was used to test the repeatability of the

Table 5 The normal reference ranges of fetal left ventricular ICT based on the cross-sectional data with the manual and automatic measurements

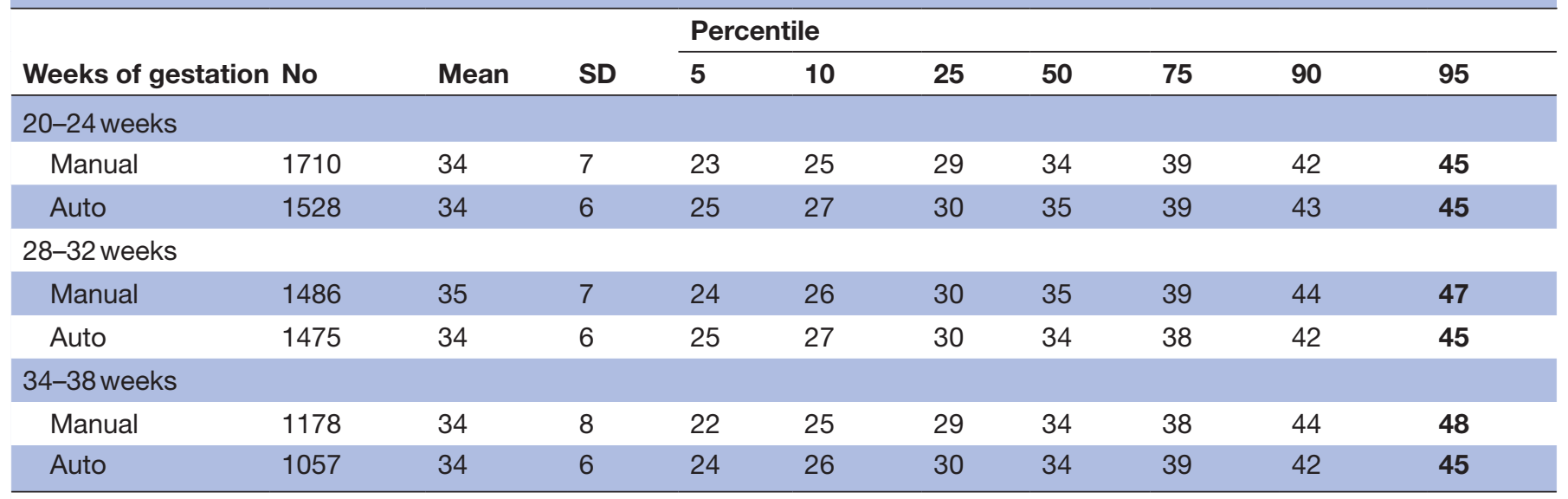

ICT, isovolumetric contraction time. 
Table 6 The normal reference ranges of fetal left ventricular ET based on the cross-sectional data with the manual and automatic measurements

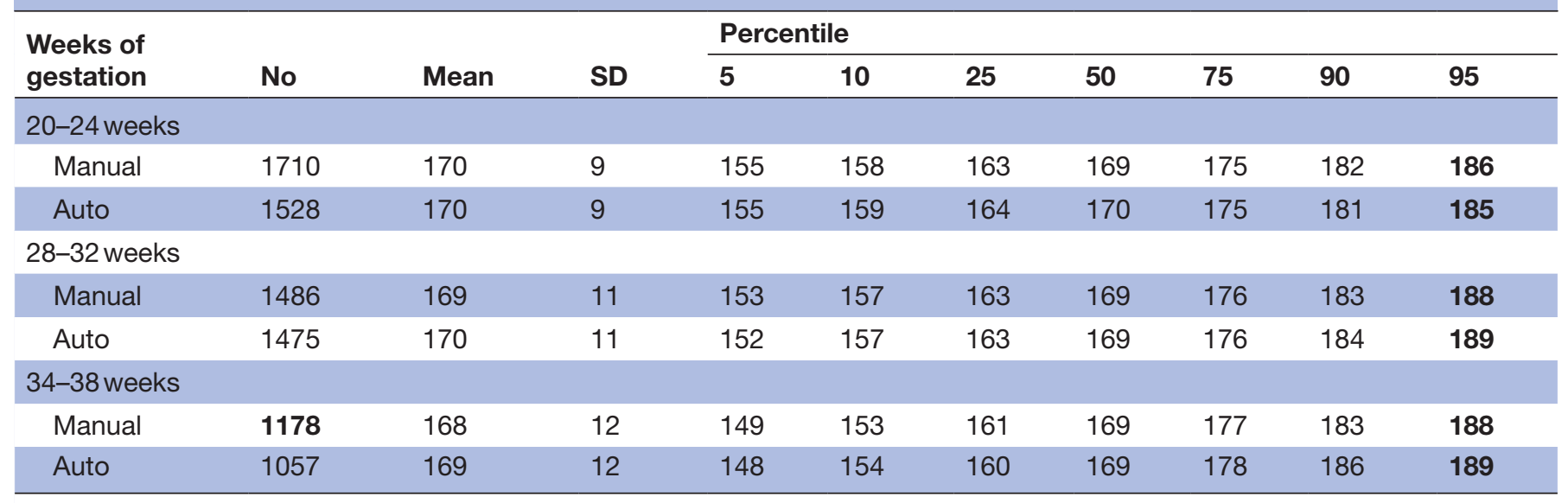

ET, ejection time.

measurement of manual and automated Mod-MPI. ICC values of less than 0.20 were considered poor; 0.21 to 0.40 , fair; 0.41 to 0.60 , moderate; 0.61 to 0.80 , good; and 0.81 to 1.00 , very good or excellent. A trend test using one-way analysis of variance was used to express the correlation between the parameters of the fetal heart function and the GAs. The best-fit regression equation was provided using linear regression models. Agreement between the manual and automated measurements was also assessed using an equivalence test. Equivalence was considered a priori if $90 \%$ CIs of the mean of the differences between the paired manual and automated measurements were within 0.01 , a clinically small difference. ${ }^{29}$ Data were analysed with SPSS V.23.0 software (IBM) and SAS V.9.4 software (SAS Institute), with statistical significance defined as $\mathrm{p}<0.05$.

\section{RESULTS}

\section{Study flow chart and the patient characteristics}

In total, 2479 women who met the eligibility criteria were recruited during $11-13^{+6}$ weeks of gestation (figure 3). Finally, 2081 fetuses were recruited in a cross-sectional study, including 465 from Beijing Obstetrics and Gynaecology Hospital, Capital Medical University, 297 from Maternal and Child Health Hospital of Hubei Province, 306 from Qingdao Women and Children's Hospital, 229 from Inner Mongolia People's Hospital, 109 from People's Hospital of Xinjiang Uygur Autonomous Region,
164 from Women's Hospital School of Medicine Zhejiang University, 148 from The International Peace Maternity and Child Health Hospital, School of Medicine, Shanghai Jiao Tong University, 286 from Shanghai Changning Maternity and Infant Health Hospital), and 77 from Shijiazhuang Obstetrics and Gynaecology Hospital. A total of 610 cases were included in the longitudinal analysis as a subgroup to test and verify the changes in fetal Mod-MPI and time intervals with advanced GA. The baseline characteristics and pregnancy outcomes of the study population are described in table 1.

The intraobserver and interobserver agreement of manual and automated Mod-MPI are shown in table 2.

\section{Relationship between parameters of fetal heart function and GA}

The Mod-MPI, IRT and ET demonstrated linear correlations with GA $(\mathrm{p}=0.001,<0.001,=0.03$, respectively), but no such correlation was seen with ICT $(p>0.05)$. A scatter plot of LV Mod-MPI, IRT and ET based on the crosssectional data showed progressive changes with advancing gestation (figure 4). The mean Mod-MPI increased slightly from 0.44 to 0.45 (Mod-MPI=0.416+0.001 $\times \mathrm{GA}$ (weeks), $\left.\mathrm{p}<0.001, \mathrm{r}^{2}=0.013\right)$. Similarly, the IRT showed a linear increase $($ IRT $=36.201+0.162 \times \mathrm{GA}$ (weeks), $\mathrm{p}<0.001$, $\left.\mathrm{r}^{2}=0.021\right)$. However, ET demonstrated a linear decrease $\left(\mathrm{ET}=171.418-0.078 \times \mathrm{GA}\right.$ (weeks), $\left.\mathrm{p}<0.001, \mathrm{r}^{2}=0.002\right)$.

Verifications for the parametric models of LV Mod-MPI, IRT and ET with advancing GA based on the longitudinal

Table 7 The agreements between the manual and automated measurements of fetal Mod-MPI obtained at 20-24, 28-32, 34-38 weeks of gestation

\begin{tabular}{lllll}
\hline Weeks of gestation & Mean & SD & Lower of $\mathbf{9 0} \% \mathbf{C l}$ & Upper of $\mathbf{9 0 \%} \mathbf{C l}$ \\
\hline $20-24$ & 0.007 & 0.040 & -0.008 & -0.005 \\
$28-32$ & 0.003 & 0.040 & -0.004 & -0.001 \\
$34-38$ & 0.004 & 0.041 & -0.006 & -0.002 \\
\hline
\end{tabular}

Mod-MPI, Modified Myocardial Performance Index. 


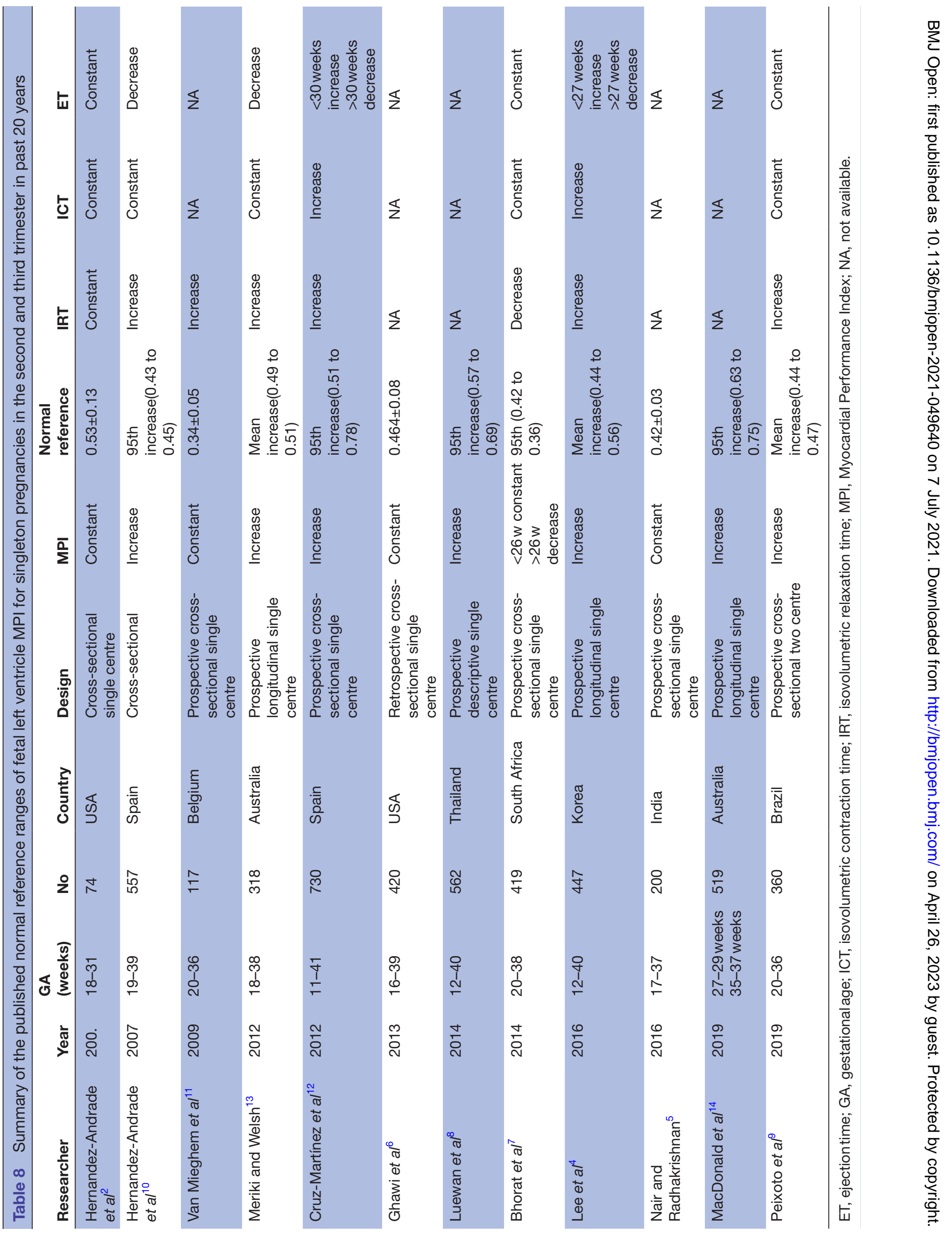


data are shown in figure 5. Similar to the cross-sectional data, the longitudinal study data also showed that ModMPI, IRT and ET had a linear correlation with GA ( $p<0.001,=0.001,0.002$, respectively), but no significant difference was seen for ICT with advancing GA ( $>0.05)$.

\section{Normal reference ranges for the parameters of fetal heart function}

The normal reference ranges for LV-Mod-MPI, IRT, ICT and ET based on the cross-sectional data with manual and automatic methods are shown in tables 3-6.

Since the inclusion was that women underwent at least one ultrasound scan at 20-24, 28-32 or 34-38 weeks of gestation, the number of patients at each GA was different and less than 2081.

\section{Agreement between the manual and automated measurements of fetal Mod-MPI}

Equivalence limits for the differences between paired manual and automated measurements were based on the clinical significance of fetal Mod-MPI. Equivalence was considered if the $90 \% \mathrm{CI}$ of the mean of the differences between the paired manual and automated measurements was within 0.01 . The agreements between the manual and automated measurements obtained at 20-24, 28-32 and 34-38 weeks of gestation were within these limits (table 7).

\section{DISCUSSION}

The normal reference ranges of fetal LV Mod-MPI by pulsed Doppler were established, and both the crosssectional and longitudinal data demonstrated a linear correlation between GA and Mod-MPI. In fact, not only previously published normal ranges of fetal Mod-MPI exhibit wide variations among studies but also the changes of fetal LV Mod-MPI with advancing weeks of gestation are conflicting $^{3-13}$ (table 8 ). Therefore, it is necessary to provide longitudinal validation of the temporal changes and normal references of fetal LV Mod-MPI to control for confounding factors. The results demonstrated that fetal Mod-MPI in the left ventricle was relatively constant throughout gestation, showing only a slight increase from 20 to 24 weeks (mean, 0.44 ; 95 th percentile, 0.52 ) to $34-38$ weeks (mean, 0.45 ; 95th percentile, 0.55 ) as pregnancy advanced. We decided a priori that the normal reference values for fetal LV-Mod-MPI would be produced from the cross-sectional data, as we hypothesised that a larger sample size of cross-sectional data would be more representative than an exclusively longitudinal study design. Prior to this study, the largest study reporting normal ranges of fetal LV Mod-MPI was from a single centre in Spain, based on cross-sectional data from 730 women. ${ }^{12}$ However, their results showed higher normal references of fetal LV-Mod-MPI, which are not applicable for the Chinese population.

Considering the small change in the time interval can lead to significant alteration in fetal LV Mod-MPI, we further analysed the changes in the time intervals in relation to GA. Throughout gestation, there was a small decrease in ET, while IRT increased slightly. ICT seemed to be constant with advancing GA. Actually, the alterations of time intervals with pregnancy advancement were inconsistent for singleton pregnancies in previous studies during the past 20 years (table 8 ). There were only three studies with longitudinal data among the twelve studies, ${ }^{41314}$ while most were cross-sectional or descriptive, as shown in table 8 . Both Meriki et $a l^{13}$ and Lee et $a t^{4}$, with longitudinal data, showed that the Mod-MPI and IRT increased with advancing GA. In addition, Meriki $e t$ $a l^{13}$ suggested that ICT was constant and ET decreased throughout gestation. These results were similar to our findings.

Our results were verified using longitudinal data from 610 women, which showed that these results were reliable. However, the rigorous definition of longitudinal cases that underwent the entire series of (three) ultrasound examinations at 11-14, 20-24, 28-32, and 34-38 weeks of gestation resulted in the sample size being relatively small.

We also demonstrated agreement between manual and automated measurements for fetal Mod-MPI. Both the mean and $95 \%$ CIs of the parameters (Mod-MPI, IRT, ICT and ET) of fetal heart function were very close using both methods of measurement. It has been previously reported that automation of fetal Mod-MPI shows excellent reproducibility and reduces the subjectivity of operators. ${ }^{23}$ Our findings suggest that this automation is reproducible and accurate with a large sample size, which means that it is a reliable technique for measuring fetal left Mod-MPI. This may allow for wider clinical application of measurements of fetal Mod-MPI due to its convenience and time savings.

\section{CONCLUSIONS}

We created normal reference ranges of fetal LV Mod-MPI by pulsed Doppler in a prospective, large, multicentre study using healthy mothers and fetuses. Automated measurement of fetal LV Mod-MPI could be considered for convenience and time savings, as there is good agreement between automated and manual values.

\section{Author affiliations}

${ }^{1}$ Department of Ultrasound, Beijing Obstetrics and Gynecology hospital, Capital Medical University, Beijing, China

'Department of Ultrasound, Qingdao Women and Children's Hospital, Shandong, China

${ }^{3}$ Department of Ultrasound, Maternal and Child Health Hospital of Hubei Province, Hubei, China

${ }^{4}$ Department of Ultrasound, Shanghai Changning Maternity \& Infant Health Hospital, Shanghai, China

${ }^{5}$ Department of Ultrasound, Inner Mongolia people's hospital, Inner Mongolia, China ${ }^{6}$ Department of Ultrasound, Women's Hospital School of Medicine Zhejiang University, Zhejiang, China

${ }^{7}$ Department of Ultrasound, the International Peace Maternity and Child Health Hospital, School of Medicine, Shanghai Jiao Tong University, Shanghai, China ${ }^{8}$ Department of Ultrasound, People's Hospital of Xinjiang Uygur Autonomous Region, Xinjiang, China 
${ }^{9}$ Department of Ultrasound, Shijiazhuang Obstetrics and Gynecology Hospital, Hebei, China

${ }^{10}$ Department of Medical Ultrasound, Taihe Hospital, Hubei University of Medicine, Hubei, China

${ }^{11}$ Department of Ultrasound, the Second Affiliated Hospital of Harbin Medicine University, Heilongjiang, China

${ }^{12}$ Department of Ultrasound, Peking Union Medical College Hospital, Chinese Academy of Medical Sciences and Peking Union Medical College, Beijing, China

Contributors All the authors are meeting the ICMJE's criteria for authorship. Substantial contributions to the conception or design of the work, drafting the work, approved the manuscript and agreed to be accountable for all aspects of the work (LS, QW, JT and YJ). Acquisition of data or analysis and interpretation of data, drafting the work, approved the manuscript and agreed to be accountable for all aspects of the work (LS, JW XS, XC, YZ, XZ, HL, JN, LY, CS, WZ, JH, LZ, ZW, PC, TC, $H H, L Z, B Y, W G, W Z, N Z, Z L$ and SZ).

Funding Beijing Municipal Administration of Hospitals'Ascent Plan, Code:DFL20151302; Beijing Municipal Science and Technology Commission (Z161100000116089)

Competing interests None declared.

Patient and public involvement Patients and/or the public were not involved in the design, or conduct, or reporting, or dissemination plans of this research.

\section{Patient consent for publication Not required}

Ethics approval This study was approved by the Institutional Review Board of Beijing Obstetrics and Gynecology Hospital, Capital Medical University (2016-KY091-01). Written informed consent to participate in the study was obtained from each patient.

Provenance and peer review Not commissioned; externally peer reviewed.

Data availability statement No data are available. Data are obtained from Beijing Obstetrics and Gynecology hospital,Capital Medical University, and are available on reasonable request.

Open access This is an open access article distributed in accordance with the Creative Commons Attribution Non Commercial (CC BY-NC 4.0) license, which permits others to distribute, remix, adapt, build upon this work non-commercially, and license their derivative works on different terms, provided the original work is properly cited, appropriate credit is given, any changes made indicated, and the use is non-commercial. See: http://creativecommons.org/licenses/by-nc/4.0/.

\section{ORCID iDs}

Lijuan Sun http://orcid.org/0000-0001-5387-8085

Qingqing Wu http://orcid.org/0000-0002-6836-9192

\section{REFERENCES}

1 Tei C, Ling LH, Hodge DO, et al. New index of combined systolic and diastolic myocardial performance: a simple and reproducible measure of cardiac function--a study in normals and dilated cardiomyopathy. J Cardiol 1995;26:357-66.

2 Hernandez-Andrade E, López-Tenorio J, Figueroa-Diesel H, et al. A modified myocardial performance (Tei) index based on the use of valve clicks improves reproducibility of fetal left cardiac function assessment. Ultrasound Obstet Gynecol 2005;26:227-32.

3 Friedman D, Buyon J, Kim M, et al. Fetal cardiac function assessed by Doppler myocardial performance index (Tei index). Ultrasound Obstet Gynecol 2003;21:33-6.

4 Lee M-Y, Won H-S, Park JE, et al. Fetal left modified myocardial performance index measured by the auto Mod-MPI system: development of reference values and application to recipients of twin-to-twin transfusion syndrome. Prenat Diagn 2016;36:424-31.

5 Nair A, Radhakrishnan S. Fetal left ventricular myocardial performance index: defining normal values for Indian population and a review of literature. Ann Pediatr Cardiol 2016;9:132-6.

6 Ghawi H, Gendi S, Mallula K, et al. Fetal left and right ventricle myocardial performance index: defining normal values for the second and third trimesters--single tertiary center experience. Pediatr Cardiol 2013;34:1808-15.

7 Bhorat I, Bagratee J, Reddy T. Gestational age-adjusted trends and reference intervals of the modified myocardial performance index (Mod-MPI) and its components, with its interpretation in the context of established cardiac physiological principles. Prenat Diagn 2014;34:1031-6.

8 Luewan S, Tongprasert F, Srisupundit K, et al. Reference ranges of myocardial performance index from 12 to 40 weeks of gestation. Arch Gynecol Obstet 2014;290:859-65.

9 Peixoto AB, Bravo-Valenzuela NJM, et al. Reference ranges for the left ventricle modified myocardial performance index, respective time periods, and atrioventricular peak velocities between 20 and $36+6$ weeks of gestation. J Matern Fetal Neonatal Med 2019;2:1-10.

10 Hernandez-Andrade E, Figueroa-Diesel H, Kottman C, et al. Gestational-age-adjusted reference values for the modified myocardial performance index for evaluation of fetal left cardiac function. Ultrasound Obstet Gynecol 2007;29:321-5.

11 Van Mieghem T, Gucciardo L, Lewi P, et al. Validation of the fetal myocardial performance index in the second and third trimesters of gestation. Ultrasound Obstet Gynecol 2009;33:58-63.

12 Cruz-Martínez R, Figueras F, Bennasar M, et al. Normal reference ranges from 11 to 41 weeks' gestation of fetal left modified myocardial performance index by conventional Doppler with the use of stringent criteria for delimitation of the time periods. Fetal Diagn Ther 2012;32:79-86.

13 Meriki N, Welsh AW. Development of Australian reference ranges for the left fetal modified myocardial performance index and the influence of caliper location on time interval measurement. Fetal Diagn Ther 2012;32:87-95.

14 MacDonald TM, Robinson AJ, Walker SP, et al. Prospective longitudinal assessment of the fetal left modified myocardial performance index. J Matern Fetal Neonatal Med 2019;32:760-7.

15 de Assunção RA, Liao AW, de Lourdes Brizot M, et al. Myocardial performance index in fetal anemia. Prenat Diagn 2015;35:192-6.

16 Henry A, Welsh AW. Monitoring intrahepatic cholestasis of pregnancy using the fetal myocardial performance index: a cohort study. Ultrasound Obstet Gynecol 2015;46:571-8.

17 Bhorat IE, Bagratee JS, Pillay M, et al. Determination of the myocardial performance index in deteriorating grades of intrauterine growth restriction and its link to adverse outcomes. Prenat Diagn 2015;35:266-73

18 Atiq M, Ikram A, Hussain BM, et al. Assessment of cardiac function in fetuses of gestational diabetic mothers during the second trimester. Pediatr Cardiol 2017;38:941-5.

19 Akkaya $\mathrm{H}$, Büke $\mathrm{B}$, Destegül $\mathrm{E}$. The effect of increased amnion volume severity on fetal Doppler indices and perinatal outcomes in idiopathic polyhydramnios. J Matern Fetal Neonatal Med 2020;33:1-7.

20 Bhorat I, Pillay M, Reddy T. Assessment of the fetal myocardial performance index in well-controlled gestational diabetics and to determine whether it is predictive of adverse perinatal outcome. Pediatr Cardiol 2019;40:1460-7.

21 Cruz-Lemini M, Valenzuela-Alcaraz B, Granados-Montiel J, et al. Characterizing cardiac dysfunction in fetuses with left congenital diaphragmatic hernia. Prenat Diagn 2018;38:422-7.

22 Cohen J, Levasseur S, Simpson L, et al. Fetal cardiac findings and hemodynamic changes associated with severe lower urinary tract obstruction in utero. Ultrasound Obstet Gynecol 2019;54:780-5.

23 Lee M-Y, Won H-S, Jeon E-J, et al. Feasibility of using auto ModMPI system, a novel technique for automated measurement of fetal modified myocardial performance index. Ultrasound Obstet Gynecol 2014;43:640-5.

24 Welsh AW, Maheshwari P, Wang J, et al. Evaluation of an automated fetal myocardial performance index. Ultrasound Obstet Gynecol 2016;48:496-503.

25 Villar J, Altman DG, Purwar M, et al. The objectives, design and implementation of the INTERGROWTH-21st project. BJOG 2013;120 Suppl 2:9-26.

26 Bhide A, Acharya G, Bilardo CM, et al. ISUOG practice guidelines: use of Doppler ultrasonography in obstetrics. Ultrasound Obstet Gynecol 2013;41:233-9.

27 Lobmaier SM, Cruz-Lemini M, Valenzuela-Alcaraz B, et al. Influence of equipment and settings on myocardial performance index repeatability and definition of settings to achieve optimal reproducibility. Ultrasound Obstet Gynecol 2014;43:632-9.

28 Meriki N, Izurieta A, Welsh AW. Fetal left modified myocardial performance index: technical refinements in obtaining pulsedDoppler waveforms. Ultrasound Obstet Gynecol 2012;39:421-9.

29 Dixon PM, Saint-Maurice PF, Kim Y, et al. A primer on the use of equivalence testing for evaluating measurement agreement. Med Sci Sports Exerc 2018;50:837-45. 